REVISTA ANDALUZA DE ANTROPOLOGÍA.

NÚMERO 10: ANTROPOLOGÍA Y EPISTEMOLOGÍAS DEL SUR: EL RETO DE LA DESCOLONIZACIÓN DE LA PRODUCCIÓN DEL CONOCIMIENTO

MARZO DE 2016

ISSN 2174-6796

[pp. 102-118]

http://dx.doi.org/10.12795/RAA.2016.10.06

\title{
ANTROPOLOGÍA Y POSCOLONIALISMO. LA MEMORIA POSTABISMAL
}

\section{ANTHROPOLOGY, POSTCOLONIALISM AND THE POST-ABYSSAL MEMORY}

\author{
Bruno Sena Martins \\ Centro de Estudos Sociais da Universidade de Coimbra
}

\section{Resumen.}

En este texto, se piensa la antropología desde una perspectiva que remite ampliamente al ámbito de la crítica postcolonial de la modernidad. Además de reconocer las asimetrías que dividieron el Norte del Sur a través de los procesos coloniales, y sobre los cuales la antropología se ha consolidado como disciplina, se trata de cuestionar cómo ese legado hoy establece desafíos para que la antropología pueda constituirse como parte de una gramática de resistencia. En este sentido, las “líneas abismales de la modernidad” (Santos, 2009) son convocadas tanto para pensar el impacto de la desmemoria colonial en los procesos sociales y políticos, como para imaginar el lugar de una antropología capaz de movilizar esa memoria a las luchas del presente.

Palabras claves: antropología, post-colonialismo, colonialismo, memoria, derechos humanos. 


\begin{abstract}
.
In this paper we reflect on anthropology from the perspective of the postcolonial critique of modernity. Recognizing the asymmetries that divided North and South through the colonial processes upon which anthropology was established as a discipline. We propose to question how this legacy imposes challenges so that anthropology can be part of resistance grammars. In that view, the "abyssal lines of modernity" (Santos, 2009) are convened both to think the impact of colonial oblivion in contemporary social and political processes, and to imagine the role of an anthropology which aims to mobilize that memory into the struggles of the present.
\end{abstract}

Keywords: anthropology; postcolonislism; colonialism; memory; human rights

\title{
1. INTRODUCCIÓN ${ }^{1}$
}

La intervención del antropólogo francés Claude Lévi-Strauss en la conferencia inaugural del Año Internacional de la Lucha contra al Racismo y la Discriminación Racial, en 1971, por invitación de la UNESCO, causó un inesperado revuelo, que el propio antropólogo señalaría más tarde, no sin ironía, como "un bello escándalo” (1983: 14).

La intención de la UNESCO al dar la palabra al reputado antropólogo en aquel contexto había sido fundamentalmente recuperar las ideas que él había presentado veinte años antes en un famoso texto titulado Race et histoire (Lévi-Strauss, 1989) y que se convertiría en una referencia en la lucha contra el racismo y contra las pretensiones de superioridad civilizacional. En ese texto emblemático, el autor sostenía como punto fuerte la necesidad de respetar y valorar la diversidad cultural en el mundo, subrayando el efecto pernicioso de los centrismos anclados en las presunciones de superioridad clamadas por ciertas formaciones culturales.

Sin embargo, no estaba en los propósitos de Lévi-Strauss replantear ideas que el tiempo había vuelto menos ciertas y que tan bien habían sido recibidas. En este sentido, en su comunicación ataca en gran medida contra "el abuso del lenguaje con que [en su opinión] se confunde cada vez más el racismo, definido en su sentido estricto, con actitudes normales,

1. Este artículo fue desarrollado en el marco del proyecto de investigación "ALICE, espelhos estranhos, lições imprevistas", coordinado por Boaventura de Sousa Santos (http://alice.ces. uc.pt) en el Centro de Estudos Sociais da Universidade de Coimbra - Portugal. El proyecto recibe financiación del Consejo Europeo de Investigación, 7 Programa Marco de la Unión Europea (FP/2007-2013)/ERC Grant Agreement no. [269807]”. 
incluso legítimas y, en todo caso, inevitables" (Lévi-Strauss, 1983: 15). En última instancia, el autor intentó alegar que las lealtades culturales, aunque en la forma de etnocentrismos susceptibles de promover indiferencia o sentimiento de superioridad respecto de otros valores, no sólo no eran necesariamente reprobables, sino que correspondían a un deseo intrínseco y legítimo de las culturas de hacer valer su singularidad. Así, la pasión por la propia cultura debería ser vista como una manera comprensible de preservación de valores que, de otro modo, estarían expuestos a la relación empobrecedora con otros sistemas culturales, mereciendo, por eso, en la visión del antropólogo, ser pensados fuera de las consideraciones condenatorias del racismo.

Creo que es importante leer el propósito de Lévi-Strauss rescatando su inclinación nostálgica sobre un mundo salpicado de culturas incontaminadas, poéticamente registrado en Tristes Trópicos (Lévi-Strauss, 1993), donde el autor vierte su desolación, lamentando la "contaminación cultural" que ya entonces marcaba la vida de los pueblos indígenas de Amazonía. Sin embargo, si es justificable, o al menos comprensible, que, ante influencias depredadoras, los grupos establezcan formas de cierre defensivo, la comprensión expuesta por Lévi-Strauss en la referida comunicación de 1971 respecto de algunas formas de etnocentrismo es un registro donde no es difícil presentir algunos peligros. Busco aquí identificar dónde, en mi opinión, emanan esos peligros.

En primer lugar, emanan del hecho de que la argumentación no se articule de un modo más efectivo con la historia y con las asimetrías en las relaciones de poder, con los imperialismos y sus epistemicidios pasados y presentes (en 1971 como ahora), no estableciendo un soporte conceptual distinto, susceptible de valorar diferentemente los movimientos opresivos y sus resistencias. Este análisis crítico del antropólogo, construido en una especie de espectralidad en relación a las realidades históricas y políticas, ha permitido, por ejemplo, que sus ideas fueran apropiadas por grupos xenófobos de los países centrales como soporte para la expulsión de los inmigrantes bajo el insólito pretexto de la defensa de la diversidad cultural de los pueblos.

En segundo lugar, porque el vislumbre de un mundo de culturas irreducibles, enamoradas de sí mismas y glorificando a sus héroes y haciendo enemigos alrededor, poco más puede augurar que la promesa tantas veces cumplida de antagonismos destructivos y tensiones irresolubles. Inscribir el pensamiento sobre las culturas y sus porosidades en las relaciones de poder y, en particular, en el colonialismo es, pues, una condición para que podamos forjar lecturas críticas inscritas en los mundos de la vida.

El largo tiempo colonial, iniciado por la expansión europea, que ha definido el sistema-mundo moderno (Wallerstein, 1974), sigue muy vivo a pesar de los procesos de independencia anticoloniales que marcaron los siglos XIX y XX. Esta persistencia se manifiesta principalmente en dos dimensiones: primero, en la continua situación de subalternidad y precariedad de las poblaciones antaño colonizadas; segundo, en la 
relación de interdependencia económica que - bien al contrario de lo que sostienen las teorías desarrollistas - hace de la existencia de una oposición entre Norte y Sur, lo local y lo global, una condición para las lógicas de acumulación del neoliberalismo.

Tanto la subalternidad como la interdependencia económica promueven un marco epistemológico que descalifica continuamente las experiencias y saberes de las personas que han sido objeto de la dominación colonial. Así, podemos hablar de pujantes legados coloniales o, utilizando el lenguaje de Ann Laura Stoller, "de formaciones imperiales": "Las formaciones imperiales son relaciones de poder. Ellas abrigan formas politicas que perduran más allá de las exclusiones formales que legislan contra la igualdad de oportunidades, las dignidades conmensurables e igualdad de derechos. Cuando se trabaja con el concepto de formaciones imperiales en lugar de imperio, el énfasis cambia de las formas fijas de soberanía y de sus negaciones a formas graduales de soberanía y a lo que ha marcado ampliamente las tecnologías de dominio imperial - escalas deslizantes y disputadas de derechos diferenciados. Las formaciones imperiales se definen por relaciones racializadas de asignaciones y apropiaciones" (Stoller, 2008: 193).

La noción de formaciones imperiales quiere poner el énfasis en los términos que la dominación imperial siempre asume y en las muchas ruinas - herencias colonialesque siguen vivas en la actualidad, ensombreciendo el futuro (Stoller, 2008: 194).

Las formaciones imperiales nos sintonizan para una disposición epistemológica bien capturada en la idea de Boaventura de Sousa Santos cuando preconiza la necesidad de "aprender con el Sur" como forma de superar la razón metonímica que la modernidad occidental ha abrazado, "la razón metonímica [es la] que se reivindica como la única forma de racionalidad y, por lo tanto, no se aplica a descubrir otros tipos de racionalidad o, si lo hace, sólo lo hace para convertirlos en materia prima" (Santos, 2002: 240).

En esencia, se trata de confrontar críticamente la soberbia civilizadora en la cual se ha asentado gran parte del discurso colonial y que todavía define gran parte de la relación de Occidente con el resto del mundo. Este es el gran reto que se plantea a las ciencias sociales en su confrontación con las experiencias, conocimientos y valores de los pueblos y sujetos oprimidos, descalificados y silenciados a la luz de las relaciones coloniales: historizar rutas sin congelarlas en el pasado; reconocer saberes e identidades sin descuidar cuánto ha sido erradicado por el colonialismo, cuánto ha sido constituido como resistencia anticolonial y cuánto se ha hibridado con la cultura del colonizador $\mathrm{y}$, finalmente, asumir que las ciencias sociales necesitan ser descolonizadas, en la medida en que se basan en las prerrogativas y privilegios científicos fundados en la hegemonía global del paradigma económico y cultural de la modernidad occidental. 


\section{VERSIONES COLONIALES DEL HUMANO}

Nacida en profunda relación con el estudio de los denominados "pueblos primitivos", la antropología, en el marco de las ciencias sociales, muy pronto se enfrentó con una paradoja matricial: al mismo tiempo que iba dando a conocer los valores y culturas que pueden cuestionar el ímpetu monocultural y civilizatorio de la modernidad, aprovechó las relaciones que, a través del colonialismo, crearon la "proximidad tóxica" a través de la cual esa misma alteridad ha podido ser conocida. Como refiere James Clifford, "de hecho, el trabajo etnográfico ha estado enredado en un mundo de desigualdades de poder duraderas y cambiables, y continúa estando implicado en ese mundo. Pero su papel en esas relaciones de poder es complejo, a menudo ambivalente, y potencialmente contrahegemónico" (Clifford, 1986: 9).

En una obra importante organizada por Talal Asad, Antropology and the Colonial Encounter (1973), se produce una reflexión sobre los vínculos entre la antropología (en particular la antropología funcionalista estructural producida en el contexto británico) y el colonialismo. Se vivía la resaca de la independencia de los territorios coloniales y una crisis disciplinaria, que ha pasado por críticas tan devastadoras como la que Peter Worsley presentó, en 1966, en una comunicación titulada significativamente The End of Anthropology (Worsley, 1970). En cuestión estaba la manera como la práctica de la etnografía antropológica, casi siempre realizada en contextos no europeos, se apoyó de tal manera en las relaciones coloniales, que ha parecido legítimo a Worsley preguntarse si el fin del colonialismo no traería el fin de la antropología. Como refleja Talal Asad: "Es indiscutible que la antropología social surgió como disciplina distinta en el inicio de la época colonial, que se convirtió en una profesión académica próspera cerca del final de esa época, o que durante este período se haya dedicado a una descripción y análisis -llevado a cabo por los europeos, para un público europeo-de sociedades no europeas dominadas por el poder europeo" (Asad, 1973: 15).

La crítica surge, en primer lugar, porque la antropología se ha desarrollado en la relación de poder entre la cultura europea (dominante) y las culturas no europeas (dominadas), relación que desde entonces ha estructurado las condiciones de posibilidad de la investigación. En segundo lugar, porque, a pesar de la ubicuidad de las relaciones coloniales, esa dimensión de la vida social estuvo a menudo omitida de los textos producidos por los antropólogos. Ausente como cuestión respecto de los temas en análisis: era posible analizar las estructuras de poder de determinado grupo social ignorando cómo la dominación colonial en ellas se entroncaba. Y ausente como elemento social, político y económico que estructura sus propias condiciones de investigación: la omisión de una reflexividad sobre el papel de las relaciones coloniales en la investigación etnográfica iba a la par de una concepción positivista del conocimiento. 
En un sentido paralelo a la reflexión de Talal Asad, sostengo que hoy la investigación en contextos postcoloniales, al silenciar las condiciones estructurales que transcurren de esa misma situación colonial, corre el riesgo de producir una omisión no menos grave que aquella que se ha apuntado al período colonial. La preocupación con el postcolonial adquiere así un sentido cercano de lo que describe Robert Young: "El postcolonial no privilegia el colonial. Está interesado en la historia colonial en la medida en que esa historia ha determinado las configuraciones y estructuras de poder del presente, en la medida en que gran parte del mundo aún vive las erupciones violentas de su despertar, y en la medida en que los movimientos de liberación anticolonial siguen siendo una fuente de inspiración de su política" (Young, 2001:4).

La cuestión candente tiene que ver, pues, no sólo con la marca histórica dejada por el encuentro colonial, sino también con una dominación cultural, económica y política que se perpetuaron de otras maneras en el período colonial: "En la actualidad, el mundo funciona dentro del sistema económico primordialmente desarrollado y controlado por Occidente, y es la dominación continuada del Occidente, en términos de poder económico, político y militar, que da a esta historia una relevancia continua. La liberación política no trajo la liberación económica -y sin liberación económica no puede haber liberación política" (Young, 2001: 5).

El pasado colonial emerge, pues, como período histórico que en mucho condiciona y determina las relaciones de poder en el presente. Recogiendo de estas estructuras labradas entre el colonialismo y las lógicas de desigualdad planetaria en que se ha venido a apoyar el capitalismo contemporáneo, la propuesta postcolonial adquiere pertinencia como una cuestión que se cruza de una manera inevitable con el análisis de contextos marcados por los despojos de la explotación económica y por la demarcación racial perpetrada por los regímenes coloniales. La lucha por el derecho a ser humano es un camino anticolonial totalmente actual.

En las sociedades cuyos conceptos de derecho y justicia se basan en una matriz eurocéntrica, encontramos repetidamente como característica una profunda omisión de la violencia colonial. Esa matriz eurocéntrica de modo alguno se reduce a Europa o a lo que vulgarmente llamamos Occidente. Por el contrario, esa matriz es muy evidente también en los lugares en que la hegemonía de los valores coloniales ha dejado por herencia relaciones de jerarquía marcadamente racializadas y monoculturalistas: " $L a$ noción de raza permite que se representen las humanidades no europeas como si fuesen un ser menor, el reflejo pobre del hombre ideal de quien estaban separadas por un intervalo de tiempo insalvable, una diferencia prácticamente insuperable" (Mbembe, 2014: 39).

El espesor histórico del colonialismo y de los procesos coloniales constituye, de hecho, una evidencia de que toda la justicia se inscribe en una historicidad que debe hacer viajar la noción de derecho fundamental a las injusticias históricas que debajo de él se ocultan. 
La gramática de los derechos humanos, cuando se considera el ser humano como dato no susceptible de discusión, independientemente de las formas de constitución de los subhumanos, es un ejemplo de eso. Así, la lucha por gramáticas de dignidad tendrá que cuestionar las formas de ser menos humano que son reproducidas por el universalismo estrecho de los derechos humanos hegemónicos (Baxi, 1989; Fanon, 2004; Rajagopal, 2006). Como afirma Achile Mbembe: "No está de más recordar que habrá sobrado algo, de las grietas e incluso de las lesiones de la crueldad colonial, para dividir, clasificar, jerarquizar $y$ diferenciar. Peor aún, la escisión creada todavía permanece" (Mbembe, 2014: 21).

El derecho a ser humano es una lucha política fundamental contra la permanencia del nexo colonial-capitalista-patriarcal y de las "prácticas de separación" (Foucault, 2003: 127) que la modernidad ha inaugurado. La consagración de denominadores mínimos de los derechos humanos, aunque congruentes con el orden global individualista, neoliberal y nortecéntrico, opera como parte de la hegemonía en la determinación de los humanos con derechos a tener derechos. La centralidad de la justicia histórica para una crítica al privilegio de ser humano con derechos se inscribe, pues, en una densa narrativa en la que la lucha por la supervivencia de la memoria no es separable de la lucha de los supervivientes que, en la actualidad, inventan gramáticas de reconocimiento y dignidad.

\section{EPISTEMOLOGÍAS DEL SUR}

Un conocimiento que enfrente las "formaciones imperiales" vigentes en el mundo y en las ciencias sociales abre la posibilidad de agendas emancipatorias que dicen tener un potencial político y epistemológico. Se trata, simplemente, de situar el conocimiento producido por la ciencia moderna dentro de las limitaciones en que se ha constituido. Por un lado, asumiendo que la mirada del investigador siempre proviene de algún lugar y nunca es una "mirada triunfante que viene de ninguna parte" (Haraway, 1998: 192). La mirada de ninguna parte que Haraway denuncia es la mirada patrocinada por una concepción positivista, a partir de la cual se niega la ubicación del sujeto que conoce, siendo este investido de una especie de "mirada de Dios" (ibid.). En segundo lugar, hay que cuestionar la neutralidad axiológica del investigador, lo que, como Boaventura de Sousa Santos defiende, no implica que se abandonen las prerrogativas de búsqueda de objetividad: "Las ciencias sociales críticas tienen, pues, que refundar una de las reivindicaciones originales de la teoría crítica moderna: la distinción entre objetividad y neutralidad. La objetividad surge de la aplicación rigurosa de métodos de investigación que nos permiten hacer análisis que no se reducen a la reproducción anticipada de preferencias ideológicas de quienes la realizan. La objetividad surge aún de la aplicación sistemática de métodos que permitan identificar los presupuestos, los prejuicios, los valores y los intereses que subyacen a la investigación científica supuestamente desprovista de ellos" (Santos, 1999: 207). 
El camino a ser recorrido por las ciencias sociales que reconocen los orígenes imperiales y positivistas de la ciencia moderna puede, en sentido contrario al de sus condiciones de origen y florecimiento, romper con las "provincias finitas del significado" (Schütz, 1970: 262) de la sociedad de partida. Este es el momento en que es posible concebir la desfamiliarización traída por la antropología como una extensión de las posibilidades críticas de la cultura occidental. Esto mismo destaca Michel Foucault cuando notó los momentos en que la etnología "suspende el largo discurso 'cronológico' por el cual intentamos reflejar nuestra propia cultura dentro de ella para hacer que aparezcan las comparaciones sincrónicas con otrasformasculturales" (Foucault, 1998:412). Aunque informe, justamente, de que el nacimiento de la etnología/antropología está profundamente inscrito en la historicidad occidental moderna donde surgió, Foucault señala cómo la etnología, lejos de enredarse en los juegos circulares de ese historicismo, terminó por romper con ellos, invirtiendo así el movimiento que la hizo nacer (1998: 409-421). Recurriendo al idioma con el cual Johanes Fabian estableció las relaciones entre etnocentrismo e imperialismo en la crítica a la antropología en Time and the Other (1983), las "comparaciones sincrónicas" de que habla Foucault corresponden a la recusación de la "negación de la coevidad": el proceso por el cual las sociedades tradicionalmente estudiadas por la antropología son sacadas de la actualidad y remitidas a un pasado primitivo, inmemorial. Sea porque la evidencia de la copresencia etnográfica tornase insostenible mantener esa paradoja fundadora, sea porque la crítica al imperialismo y a sus trabas metodológicas ganase justo lugar; el momento que aquí se comenta es, pues, el de la aceptación de la coevidad en términos que la acercan a las "epistemologías del Sur" (Santos, 2014). A través de las epistemologías del Sur, Boaventura de Sousa Santos propone una reflexión sobre los desafíos de la emancipación en un tiempo en que "un sentido de agotamiento asombra la tradición crítica occidental, eurocéntrica” (Santos, 2014: 19). En ese sentido, se propone una crítica de la modernidad occidental con base en una "teoría de retaguardia" (ibid.: 11: 44) capaz de captar la "diversidad [inagotable] del mundo" (ibid.: 108). El autor identifica cuatro estrategias analíticas para la constitución de las epistemologías del Sur. En primer lugar, la "sociología de las ausencias" propone la visibilización de conocimientos que las ciencias sociales dominantes han vuelto irrelevantes o inexistentes.

En segundo lugar, la "sociología de las emergencias" es "la investigación de las alternativas que caben en el horizonte de las posibilidades concretas", forjando un futuro de posibilidades plurales y de expectativas más amplias (Santos, 2014: 184).

En tercer lugar, la ecología de saberes "enfrenta la lógica del monocultivo del saber científico $y$ del rigor identificando otros conocimientos $y$ otros criterios de rigor y validez que operan de forma creíble en las prácticas sociales" (Santos, 2014: 188). La "ecología" subraya la coexistencia de diversas formas de conocimiento como una relación compleja que se basa en el reconocimiento mutuo. En consonancia con la "ecología de los saberes, la cuarta 
forma de investigación es la traducción intercultural, a través de la cual se constituyen 'nuevas formas híbridas de entendimiento e intercomunicación cultural"' (Santos, 2014: 212).

\section{VOCES SUBALTERNAS}

La propuesta de las "epistemologías del Sur", en mi opinión, da una justa réplica a la cuestión de Gayatri Spivak (1999): Can the subaltern speak? [¿Puede el subalterno hablar?]. O sea, las voces subalternadas por el imperialismo de la razón metonímica moderna occidental hablan en la medida que puedan determinar sus destinos y, simultáneamente, puedan desafiar las premisas de las lógicas opresoras.

La pregunta de Spivak proviene del análisis que la autora realiza sobre la situación de las mujeres hindúes en la lucha anticolonial. La reflexión de Spivak parte de las cuestiones planteadas en torno del ritual de inmolación de las viudas (Sati) para mostrar cómo la situación de las mujeres queda ahí irónica y trágicamente expuesta: "Entre la formación patriarcal del sujeto y la constitución imperialista del objeto, es el espacio de libre albedrío de los sujetos constituidos como mujeres el que es borrado con éxito" (Spivak, 1999: 235).

El Sati era un ritual que llevaba a la muerte por el fuego a las viudas y que era practicado principalmente en la región de Bengala. Esta práctica, si bien no constituía una obligación inequívoca, era a menudo llevada a cabo por las viudas, puesto que su realización era socialmente sancionada por la lógica patriarcal dominante. El gobierno colonial británico entendió prohibir esta práctica como forma de liberar a las mujeres de la tiranía indígena. Al prohibir la práctica del Sati, este ritual adquirió un significado de transgresión congruente con la resistencia anticolonial. Por consiguiente, en la toma de decisión de las viudas ante la opción de autoinmolación, surge una nueva descripción posible para su acción, el Sati se constituye como un "significante excepcional" contrahegemónico. Por lo tanto, las viudas se encontraban ante la posibilidad de suicidarse ritualmente, en un acto que pasa a ser entendido principalmente como una forma de disidencia; o seguir vivas cumpliendo la prohibición impuesta por las autoridades coloniales. En esta situación dilemática, cualquiera de las posibilidades niega la subjetividad de las mujeres: o porque se ratifica un patriarcado invertido en una lucha contra la potencia ocupante, o porque se acepta la aspiración colonialista de domar la alteridad del otro colonizado. Por lo tanto, hay aquí dos narrativas posibles en que las mujeres hindúes pueden tomar parte en la lucha por el significado, pero donde la representación de sus voces fracasa en encontrar lugar. La muerte como significante excepcional y puerta de acceso a una narrativa disruptiva es la imagen más poderosa de esa negación.

La lectura de Spivak, reificada en el análisis del Sati, expresa bien en qué medida ciertas oportunidades de transformación social contrahegemónica producen, también ellas, 
formas de silenciamiento y descalificación. Así, la pregunta planteada por Spivak -Can the Subaltern Speak? - busca problematizar al subalterno, en tanto que diferente del oprimido, como alguien que no puede/consigue hablar. El "hablar", bajo los términos en que Spivak refleja, implica ser escuchado, y se dirige hacia la eventual diferencia que la enunciación de una voz puede o no hacer. La formulación de Spivak no permite distinguir silencio de silenciamiento. De hecho, este tema no es suficientemente problematizado por la autora. Pero, al construir el subalterno en la ambigüedad entre aquel a quien no dejan hablar y aquel que no tiene las condiciones de posibilidad para la enunciación de su voz, la autora expresa una sensibilidad posestructuralista que nos envía a ese doble momento de las relaciones de poder. Por un lado, la autoridad para silenciar y descalificar voces y, por otro, la siempre discutible capacidad de definir qué enunciaciones pueden ser articuladas en un determinado momento socio-histórico.

Por consiguiente, la valoración de las voces subalternas se concilia con el proyecto de "provincializar Europa", propuesta enunciada por Dipesh Chakrabarty (2000) para referir la necesidad de revertirse el nexo universalista e imperialista del Occidente: "Llamémosle el proyecto de provincializar 'Europa', la Europa que el imperialismo moderno y que el nacionalismo (del tercer mundo), por su empresa y violencia, han hecho universal" (Chakrabarty, 2000: 42).

La formulación de Dipesh Chakrabarty no sostiene la recusación apriorística de los productos epistemológicos de origen europeo (en el caso relacionados con la historia), a la luz de un relativismo ingenuo; lo que hace es desenmascarar el efecto hegemónico pernicioso de las prerrogativas de verdad alcanzadas por los cánones de la razón iluminista: "Porque el argumento no es que el racionalismo del Iluminismo sea siempre irrazonable en sí, sino más bien una cuestión de documentar cómo - a través de qué proceso histórico-su 'razón', que no ha sido siempre autoevidente para toda la gente, se ha hecho obvia mucho más allá del campo donde se originó. Si una lengua, como se ha dicho, no es más que un dialecto apoyado por un ejército, lo mismo puede decirse de las narrativas de la modernidad que, hoy casi universalmente, apuntan hacia una cierta 'Europa' como el habitus primordial de lo moderno" (Chakrabarty, 2000: 43).

\section{CULTURAS: ENCUENTROS Y FRONTERAS}

Boaventura de Sousa Santos afirma que todas las culturas son incompletas y problemáticas en sus concepciones de dignidad humana, y que la idea de completitud es un exceso de sentido del que parecen padecer todas las culturas, siendo que la conciencia necesaria de incompletitud podrá ser incrementada al acceder a la perspectiva de otras culturas a través de una retórica dialógica (tercera premisa) (Santos, 1997: 22). 
Partiendo de esta idea, me parece importante que se comprenda que el proceso histórico de constitución de formaciones culturales que reivindican su completitud, y elaboran su cierre, sucede en el propio proceso de interacción con otras culturas. Con eso, prestamos atención a la manera como las identidades son constituidas relacionalmente y formadas en el proceso de elaboración de la diferencia.

Para esta sedimentada persuasión nos conduce el análisis influyente que Frederik Barth (1981) produce en Ethnic groups and boundaries. Aunque la visión del autor padezca aún de una perspectiva anclada de las culturas poco sensible a los procesos de interpenetración, en una imaginación territorial donde se consagra la frontera como zona de contacto casi exclusivo, su cuestionamiento no deja de ser pertinente al denunciar la insostenibilidad de las nociones de diversidad cultural que persisten en el hecho de pensar los grupos en la ignorancia de sus vecinos. Así, el autor afirma que "las distinciones étnicas no dependen de la ausencia de interacción social y de aceptación, sino, al revés, son a menudo los fundamentos donde se construyen los sistemas sociales" (Barth, 1981: 199). En última instancia, se sostiene que toda identidad está fuera de sí misma. Esta lectura permite desde luego un distanciamiento en relación a una cierta visión seductora y romántica que solía colocar la aparición de la creatividad cultural en un aislamiento prístino de las culturas. De aquí resulta que al pensar las culturas investidas en una "hermenéutica diatópica" (Santos, 1997), o en una traducción intercultural (Santos, 2014), hay que reconocer en qué medida las culturas y las formaciones culturales son ya productos de hermenéuticas dialógicas, a menudo constituidas bajo el espectro del antagonismo.

Esta manera de pensar la constitución de formaciones culturales deberá tener como virtualidad fuerte centrar nuestra atención menos en el aumento de la conciencia de incompletitud que surge de la relación diatópica con el Otro y más en la transformación del carácter de la relación con el otro en un sentido diatópico. Es decir, la traducción intercultural deberá ser incentivada menos como la inauguración de una relación de aprendizaje y más como la propuesta de una relación que se quiere nueva, en el sentido de otro modo de producción de conocimiento.

Si bien es cierto, como afirma Boaventura de Sousa Santos, que vivimos en una época de porosidades (Santos, 2000: 224), parece igualmente claro que vivimos en un tiempo de recolocación dinámica de las fronteras, donde las comunidades de intercambio y aprendizaje intercultural ocupan aún un lugar residual. Aparente paradoja, ilustrativamente lograda en situaciones de desigualdad e impermeabilización mutua que marcan los procesos migratorios contemporáneos.

En realidad, el carácter relacional de las culturas recibe hoy merecida atención ante un mundo cada vez más marcado por profundas interpenetraciones a diversos niveles, bajo los auspicios del colonialismo de las prácticas capitalistas globales. Por eso, el momento contemporáneo hace que sea todavía más importante una sensibilidad capaz de entender 
las culturas, no sólo en relación al papel constitutivo de las vecindades históricas y geográficas, sino también por referencia a la vigencia actual de una hegemonía global, establecida en la intensificación de la expansión económica y cultural de Occidente. Y, si bien es cierto que las profecías de homogeneización cultural y elisión de la diferencia en el mundo están lejos de cumplirse, lo que parece igualmente inevitable es el hecho de que la persistencia de las constelaciones de las diferencias se defina en la intimidad con la globalización promovida por las prácticas del capitalismo global. Este tema es tratado por Wallerstein cuando intenta afirmar en qué medida la intensificación de las relaciones de la economía-mundo se dio en concomitancia con la constitución de diferenciaciones culturales profundamente inscritas en las relaciones de poder de la modernidad: " $L o$ que es sorprendente acerca de la historia politica del moderno sistema-mundo, en la forma como se desarrolló históricamente, es el uso cada vez más frecuente y efectivo por parte de los elementos oprimidos de lo que genéricamente se podrían llamar culturas de resistencia" (Wallerstein, 1991: 99).

En consecuencia, el autor da cuenta de cómo se inscribe, como característica de la formación del sistema-mundo moderno, la definición de fronteras y de culturas en procesos eminentemente políticos y económicos de opresión y resistencia. En el mismo sentido sigue el análisis de Arjun Appadurai (1998) cuando aborda las estrategias discursivas de lo que designa por "culturalismos", estrategias que según el autor impregnan los procesos de formación de la diferencia cultural en la modernidad. Según Appadurai, el concepto de culturalismo comprende la movilización consciente de las diferencias culturales al servicio de afirmaciones identitarias en las formaciones nacionales y transnacionales. Al pensar en los modos de producción y reproducción de la diferencia en la modernidad, Appadurai intenta captar, con el concepto de culturalismo, la medida en que éstos ocurren en construcciones deliberadas, estratégicas, populistas, evocando el autor la paradoja singular del "primordialismo construido" como una de sus expresiones. En este sentido, el autor no podría ser más enfático cuando dice: “culturalismo es la forma que las diferencias culturales tienden a tomar en la era de los medios de comunicación de masas, de las migraciones y de la globalización" (Appadurai, 1998: 16).

\section{LA VIOLENCIA COLONIAL Y LA MEMORIA ABISMAL}

Al reflexionar sobre la historia y sobre el archivo, Marc Bloch señala que varias sociedades practicaron lo que podríamos llamar "bilingüismo jerárquico" (2010). Se trata de la coexistencia de dos lenguas, una popular y otra de estudio; la primera utilizada para pensar y hablar, la segunda utilizada sobre todo en la escritura. De los varios ejemplos que Bloch refiere, destaco el modo como los evangelistas registraron en griego conversaciones que habrán ocurrido en arameo o el modo como el latín fue utilizado en la Edad Media como idioma en que eran registrados los procedimientos administrativos y las narrativas 
relevantes. Esta coexistencia jerárquica, sostiene Bloch, implica que los escribas creen un archivo al mismo tiempo que realizan una transposición entre idiomas 冈es decir, una transposición entre el idioma de la experiencia y el idioma oficial (más afín de la historia oficial). Sabiéndose que el idioma del archivo es el que mejor resiste al tiempo, se percibe el aporismo que se plantea al historiador: una sociedad conocida a través de los registros escritos será inevitablemente sesgada a la mirada del historiador por el velo que resulta de la transposición entre los dos idiomas (Bloch, 2010: 136).

En mi opinión, la asimetría creada por el bilingüismo jerárquico sólo se puede superar mediantela superación de las “líneas abismales de la modernidad”. Al definir el pensamiento moderno como abismal, Boaventura de Sousa Santos enfatiza como todavía persisten los mecanismos que, separando sociedades metropolitanas y territorios coloniales, descalifican e invisibilizan las experiencias y saberes considerados como inmanentes a la zona colonial (Santos, 2009). En este sentido, se generan "distinciones invisibles [que] son establecidas a través de líneas radicales que dividen la realidad social en dos universos, el universo de "este lado de la línea" y el universo del "otro lado de la línea". La división es tal que "el otro lado de la línea" desaparece como realidad, se convierte en no existente, y de hecho es producido como no-existente. No-existente significa no existir en ninguna forma relevante o comprensible de ser" (Santos, 2009: 31).

En consonancia con la propuesta de Boaventura de Sousa Santos, creo que podemos hablar de una memoria abismal para caracterizar cómo la experiencia colonial, en lo que tiene de más característico — la violencia que la sostiene-, está ausente de las historias que constituyen, en el Norte, las narrativas sobre el "otro lado de la línea”. Es esta memoria abismal la que permite que tanto en el sentido común como en las ciencias sociales prevalezcan lecturas en que la violencia colonial es silenciada o doblada a los mitos fundacionales que expurgan el sufrimiento producido por la vía colonial, reniegan la permanencia de sus lógicas y producen la no-existencia de los sujetos que la atestiguan. La valoración del sufrimiento sigue líneas raciales que sacan del tiempo a los sujetos que experimentaron y experimentan en su constitución ontológica lo que hay de inevitable en la violencia colonial.

\section{UNA MEMORIA POSTABISMAL: PARA DESCOLONIZAR EL PRESENTE}

Una concepción viajada de dignidad humana tendrá que constituirse en traducciones conscientes de las grandes desigualdades establecidas por la historia colonial y por la arrogancia monumental de los edificios de la ciencia moderna. La búsqueda del subalterno es tanto la posibilidad de reconocer la voz a sujetos existentes, actualmente marginados y desposeídos, como la constitución de nuevos sujetos a través de la traducción intercultural, sea para transformar el lugar y el ser a quienes se niegan a 
abandonar las posiciones de privilegio, o para construir nuevas posibilidades de autoría social entre aquellos que hace mucho tiempo reclaman la urgencia de otros mundos posibles. La necesidad de construcción de idiomas de dignidad humana entre culturas deberá conducir a la producción de culturas viajadas en viaje a lugares de emancipación social; de hecho, necesitamos nuevos viajes que puedan atravesar las fronteras hace mucho tiempo implantadas en los rincones del mundo colonial en que vivimos.

Una forma especialmente instigadora de formular el compromiso de la antropología en un mundo postcolonial es entender las experiencias marcadas por las formaciones imperiales como narrativas de opresión y resistencia que, a su modo, pueden orientar el trabajo de investigación hacia una insurgencia inspirada en ellas. Yo diría que estamos ante resistencias cuyas afinidades nos remiten a la primera de las cuatro formas de semejanza que Michel Foucault distingue en Les mots et les choses (1998), la convenientia, una similitud que nace de la proximidad. Como escribe el autor: "Son 'convenientes' las cosas que, acercándose unas a las otras, llegan a confinar; se tocan en los bordes, sus fimbrias se mezclan, la extremidad de una señala el comienzo de la otra. De esta manera, el movimiento se comunica, como las influencias, las pasiones y las propiedades. De suerte que en la bisagra de las cosas surge una semejanza. Después, de ese contacto nacen, por intercambio, nuevas semejanzas; se impone un régimen común; a la semejanza como razón sorda de vecindad se superpone una semejanza que es el efecto visible de la proximidad" (Foucault, 1998: 74).

Una antropología válida es aquella que, por la "semejanza de la proximidad", por la partición de "influencias", "pasiones" y "propiedades", por el aprendizaje con las resistencias situadas, asume la lucha por una sociedad incluyente como parte de una objetividad fuerte, construida lado a lado con las agendas anticoloniales. Las historias de violencia colonial se mueven dentro de esquemas de inteligibilidad que establecen hábitats poblados por modos estrechos de concebir la experiencia. La lucha por la dignidad humana no es pensable fuera de la lucha entre los edificios de la ciencia moderna occidental y del colonialismo que fueron su contraparte. La antropología, no sin paradoja histórica, será tanto más válida cuanto rechace la memoria abismal para producir y ser parte de lo que designo como memoria postabismal.

Tomando la noción de "pensamiento abismal" de Boaventura de Sousa Santos (2009, 2014), identifico dos sistemas de significado que velan por la experiencia colonial en sentidos opuestos: la memoria abismal y la memoria postabismal. La memoria abismal constituye un sistema de significado, dominante, en el cual, durante décadas, la violencia colonial fue ostensiblemente borrada, silenciada y empujada hacia el olvido. Este sistema de significado es aquel que se concierta con las representaciones de una Europa moldeada por una autorrepresentación benevolente, que extirpa la inmanencia política, económica y cultural del colonialismo de la memoria de la modernidad occidental. Según expone 
Eduardo Lourenço: "El abordaje crítico a la complejidad esfíngica de Europa continúa encubriendo la necesidad de una descolonización completa del pensamiento y de la imaginación hegemónicos de Europa, o sea, de la necesidad de una efectiva descolonización de Europa de su propia experiencia colonial" (Lourenço, 2014: 16). Es esta memoria abismal la que consiente historiografías nacionales en que la relación con el Sur global es silenciada o plegada a los mitos fundadores que, a un tiempo, expurgan el sufrimiento producido por la senda colonial, reniegan la permanencia de sus lógicas y producen la no existencia de los sujetos que las atestiguan.

La memoria postabismal, alego, es aquella cuya mirada hacia el pasado colonial inscribe la violencia colonial en el centro de la narrativa histórica de la modernidad. La memoria postabismal es aquella que identifica la memoria abismal como un segundo aliento de la violencia colonial y, al rechazarla, denuncia una violencia que racializa el valor de la vida. Una memoria postabismal de la violencia tendrá que ser capaz de abrazar las vastas latitudes de la experiencia moderna, los vastos sures, cargando los cuerpos y la violencia, muertos y sobrevivientes, reconstituyendo a Norte y Sur los fragmentos de una violencia que está lejos de haber terminado. 


\section{REFERENCIAS BIBLIOGRÁFICAS}

Appadurai, Arjun (1998) Modernity at Large: Cultural Dimensions of Globalization. Minneapolis: University of Minnesota Press.

Asad, Talal (coord.) (1973) Anthropology \& the Colonial Encounter. Nueva York: Humanity Booksed.

Barth, Frederik (1981) Process and Social Form in Social Life. Londres: Routledge \& Kegan Paul.

Baxi, Upendra (1989) "From Human Rights to the Right to be Human: Some Heresies". En Smitu Kothari y Harsh Sheth (coords.) Rethinking Human Rights. Delhi: Lokayan, pp. 151-167.

Bloch, Marc (2010) The historian's craft. Manchester: Manchester University.

Chakrabarty, Dipesh (2000) Provincializing Europe: postcolonial thought and historical difference. Princeton, N.J.: Princeton University Press.

Clifford, James (1986) “Introduction: Partial truths". En James Clifford y George E. Marcus (coords.) Writing Culture: The poetics and politics of ethnography. Berkeley: University of California Press, pp. 1-25.

Fabian, Johanes (1983) Time and the Other: How Anthropology Makes its Object. Nueva York: Columbia University Press.

Fanon, Frantz (2004) The wretched of the earth. Nueva York: Grove Press.

Foucault, Michel (1998 [1966]) As Palavras e as Coisas. Lisboa: Edições 70.

Foucault, Michel (2003) “Security, Territory, and Population”. En Paul Rabinow y Nikolas Rose (coords.) The Essential Foucault: Selections from Essential Works of Foucault, 19541984. Nueva York: New Press, pp. 259-262.

Haraway, Donna (1998) “The Persistence of Vision". En Nicholas Mirzoeff (coord.) The Visual Culture Reader. Londres: Routledge, pp. 677-684.

Mbembe, Achile (2014) A Crítica da Razão Negra. Lisboa: Antígona.

Lévi-Strauss, Claude (1983) O Olhar Distanciado. Lisboa: Edições 70.

Lévi-Strauss, Claude (1989 [1952]) Raça e História. Lisboa: Editorial Presença.

Lévi-Strauss, Claude (1993) Tristes Trópicos. Lisboa: Edições 70.

Lourenço, Eduardo (2014) Do Colonialismo como Nosso Impensado. Lisboa: Gradiva.

Rajagopal, Balakrishnan (2006) "Counter-Hegemonic International Law: Rethinking Human Rights and Development as a Third World Strategy". Third World Quarterly 27(5), pp. 767-784. 
Santos, Boaventura de Sousa (1997) "Por uma Concepção Multicultural de Direitos Humanos”. Revista Crítica de Ciências Sociais, 48, pp. 11-32.

Santos, Boaventura de Sousa (1999) Pela Mão de Alice: O Social e o Político na PósModernidade. Porto: Afrontamento.

Santos, Boaventura de Sousa (2000) Crítica da Razão Indolente: Contra o Desperdício da Experiência. Porto: Afrontamento.

Santos, Boaventura de Sousa (2002) "Para uma Sociologia das Ausências e uma Sociologia das Emergências". Revista Crítica de Ciências Sociais, 63, pp. 237-280. Disponible en http://rccs.revues.org/1285.

Santos, Boaventura de Sousa (2009) "Más allá del pensamiento abismal: de las líneas globales a una ecología de saberes". En Luís Tapia Mealla (coord.) Pluralismo Epistemológico. La Paz: CLACSO/CIDES-UMSA/Muela del Diablo/Comuna, pp. 31-84. Santos, Boaventura de Sousa (2014) Epistemologies of the South: Justice Against Epistemicide. Boulder: Paradigm Publishers.

Schütz, Alfred (1970) On Phenomenology and Social Relations: Selected Writings. Chicago: University of Chicago Press.

Spivak, Gayatri (1999) A Critique of Postcolonial Reason: Toward a history of the vanishing present. Cambridge, M.A.: Harvard University Press.

Stoller, Ann Laura (2008) "Imperial Debris: Reflections on ruins and ruination". Cultural Anthropology, 23(2), pp. 191-219.

Wallerstein, Immanuel (1974) The Modern World-System [Vol.1]. Nueva York: Academic Press.

Wallerstein, Immanuel (1991) "The National and the Universal: Can there be such a thing as World Culture?". En A. King (coord.) Culture Globalization and the World System. Londres: Macmillan, pp. 91-105.

Worsley, Peter (1970) “The End of Anthropology”. En Transactions of the Sixth World Congress of Sociology (vol. 3). Lovaina: International Sociology Association, pp. 121-129. Young, Robert (2001) Postcolonialism: An Historical Introduction. Oxford: Blackwell. 